\title{
Technology review
}

\section{The 3D Static Strength Program ${ }^{\mathrm{TM}}$}

\author{
Deborah Lechner
}

The 3D Static Strength Program ${ }^{\mathrm{TM}}$ (3DSSPP) is one of several computer software programs developed by the Centre for Ergonomics at the University of Michigan. Like the previously reviewed 2D Static Strength Program (2DSSPP), the 3DSSPP is useful in evaluating, and modifying manual materials handling tests. The program allows the user to make predictions regarding the relative risk of a task based on the posture, exertion, and anthropometry of the worker. In contrast to the 2DSSPP, which can be used only to evaluate tasks that occur in the sagittal plane, the 3DSSPP simulates and evaluates tasks in three-dimensional space. Such analysis is helpful in analysing tasks that involve torso rotation and bending. In addition, the 3DSSPP allows evaluation of one-hand exertions, pushing and pulling.

The newly released version 2.0 of the 3DSSPP runs on Microsoft Windows with pull-down menus and requires an IBM-PC or compatible with a 486 microprocessor and math co-processor and clock speed of $33 \mathrm{MHz}$. The program also requires a VGA monitor and mouse, Microsoft Windows 3.1 running in enhanced mode, $4 \mathrm{MB}$ RAM, and 1 $\mathrm{MB}$ of available hard drive disk space. The new graphics and improved algorithms offers an improvement in accuracy and efficiency over version 1.0. A 10 -min video that demonstrates some of the features of the software is available for a $\$ 15$ charge. The program costs $\$ 1000$ plus shipping for the standard first-time license of $\$ 150$ for an upgrade from version 1.0.

The program is designed such that the user enters the information regarding the magnitude and direction of the force exerted, anthropometry of the worker, and posture information. Posture information can be entered using any one or a combination of two approaches. Joint angles can be entered directly as measured from the photograph of videotape. Alternatively, the worker's hand location can be entered relative to the feet. Given the hand location, the program will automatically calculate the joint angles and generate a 'predicated' posture. The 'predicted' posture can then be modified by using the pull-down menus and mouse to change joint angles until the graphic most closely represents the actual posture being studied. The user can also use this point and click method of modifying the graphic or enter changes in joint angles to determine the effect of those changes on the various outputs.

The software generates three-dimensional figure illustrations that can be used to verify the accuracy of the posture being modeled by comparing the figure to the video/photo. The program offers three choices of figure graphics; the stick figure, a wire mesh figure superimposed over the stick figure, and a fully faceted figure. The figures are displayed in three orthogonal views; front, side, overhead, and a user-defined oblique 
view. This oblique view assists the user in comparing the modeled posture to the video/photo. The figures are drawn to scale and include force vector direction arrows originating from the hands. The program also offers the user the option of including hand-held objects, barriers, scat, and floor in the graphic display. The graphic can be imported into other documents which can be used for reports and presentations.

In addition to the figures, the program outputs include: $15 /$ Sl disc compression and shear forces, L2/3 through L4/5 moments and forces, resultant joint moments and relative strengths, comparison of the predicted forces with NIOSH guidelines, body link lengths and masses, status of body balance, 3D optimization for compression force at L4/5 disc and torso muscles and static coefficient of friction required to prevent foot slippage.

Comments regarding this program were made based upon evaluation of a demo disc provided by the University of Michigan. In general, the program seems to be powerful yet fairly intuitive and user friendly. For the most part, screens were well-designed and easy to interpret. Menu choices were clearly labelled and dialog boxes easy to use. The program, however, is not meant for the novice but requires an understanding of biomechanics and job analysis in addition to understanding the program. The user also must be familiar with use of a mouse and pull-down menus. Excerpts from the procedure manual suggest that the manual is well-written and organized for the case in use. As previously noted in the 2DSSPP review, the technical staff are helpful in addressing any problems you may encounter. The demo ran smoothly and quickly on a hardware system configured as described above. The only criticism this reviewer found with the program was that the output screens and printouts were not very sophisticated in appearance. These output screens and printouts are a sharp contrast to the high-tech figure graphics included in the program. Accord ing to University of Michigan, the output screens / printouts are low-tech in appearance due to the many printer interface problems encountered by their users when attempting to print the bar graphs included in their earlier high-tech printouts. This aesthetic problem with the output is not a major problem in an otherwise excellent and powerful program. However, in an increasingly competitive market, ergonomists must consider the appearance and user-friendliness of their reports. University of Michigan staff stated that they would be addressing this problem in future versions. 